\title{
APPL1 ameliorates myocardial ischemia-reperfusion injury by regulating the AMPK signaling pathway
}

\author{
YUNGUANG CEN $^{1}$, WEI LIAO ${ }^{2}$, TAIHAO WANG ${ }^{1}$ and DAIMIN ZHANG ${ }^{3}$ \\ ${ }^{1}$ Department of Geriatric Center and ${ }^{2}$ Department of Medical Ultrasonics, \\ Hainan General Hospital (Hainan Affiliated Hospital of Hainan Medical University), Haikou, Hainan 570311; \\ ${ }^{3}$ Department of Cardiology, Nanjing First Hospital, Nanjing Medical University, Nanjing, Jiangsu 210006, P.R. China
}

Received December 11, 2020; Accepted September 1, 2021

DOI: $10.3892 / \mathrm{etm} .2021 .11080$

\begin{abstract}
Myocardial ischemia-reperfusion injury results in elevated reactive oxygen species (ROS) production and causes oxidative stress damage. Therefore, the current study aimed to investigate whether adaptor protein phosphotyrosine interacting with $\mathrm{PH}$ domain and leucine zipper 1 (APPL1) could induce the expression of antioxidant enzymes through AMP-activated protein kinase (AMPK) signaling in order to alleviate the injury caused by ischemia/hypoxia-reperfusion. Following induction of hypoxia-reoxygenation $(\mathrm{H} / \mathrm{R})$ injury in $\mathrm{H} 9 \mathrm{c} 2$ cells, the liver kinase B1 (LKB1)/AMPK/acetyl-CoA carboxylase $\alpha$ (ACC) signaling pathway was investigated using western blot analysis, along with the detection of superoxide dismutase (SOD)2 and SOD3 expression. Additionally, cell viability was detected using a Cell Counting Kit-8 assay and ROS production was analyzed using ROS staining, whereas the expression levels of inflammatory mediators (TNF- $\alpha$, monocyte chemoattractant protein 1 and IL-1 $\beta$ ), apoptosis mediators [cleaved caspase-3, cleaved poly (ADP-ribose) polymerase and Bcl-2] and nuclear factor erythroid 2-related factor 2 signaling pathway-related proteins were detected via western blot analysis following overexpression of APPL1 alone or in combination with compound $\mathrm{C}$ treatment (an AMPK inhibitor). The results indicated that $\mathrm{H} / \mathrm{R}$ induction upregulated the phosphorylation levels of LKB1, AMPK and ACC, and decreased the expression levels of APPL1 and SOD enzyme activities. APPL1 overexpression increased the phosphorylation levels of LKB1, AMPK and ACC, SOD enzyme activity and cell viability whereas the expression
\end{abstract}

Correspondence to: Dr Daimin Zhang, Department of Cardiology, Nanjing First Hospital, Nanjing Medical University, 68 Changle Road, Nanjing, Jiangsu 210006, P.R. China

E-mail: zhangdaiyuandy@outlook.com

Key words: adaptor protein phosphotyrosine interacting with $\mathrm{PH}$ domain and leucine zipper 1, myocardial ischemia-reperfusion, AMP-activated protein kinase, nuclear factor erythroid 2-related factor 2 levels of proinflammatory mediators and proapoptotic mediators, and the levels of ROS production were markedly decreased when compared with H/R group with empty plasmid transfection. APPL overexpression-mediated effects were significantly abrogated by compound C. Taken together, the data indicated that APPL1 inhibited ROS production and $\mathrm{H} / \mathrm{R}$-induced myocardial injury via the AMPK signaling pathway. Therefore, APPL1 may serve as a potential therapeutic target for myocardial H/R injury.

\section{Introduction}

Acute myocardial infarction (AMI) is a cardiovascular disease with common and potentially fatal presentation (1). Although there was a relative decline in AMI of $48.7 \%$ over a study period (2000-2014), AMI still is a leading cause of morbidity and mortality worldwide (2). The pathogenesis of myocardial ischemia-reperfusion ( $\mathrm{I} / \mathrm{R})$ injury is considered one of the main research topics in the field of vascular disease $(3,4)$. The mechanism underlying myocardial I/R injury has not been fully elucidated. $\mathrm{Ca}^{2+}$ overload and oxidative stress are the primary factors associated with this disease, and inflammatory immune responses and induction of apoptosis are also involved $(5,6)$.

During I/R, AMP-activated protein kinase (AMPK) signaling serves a role in oxidative stress, cell apoptosis and cardiac dysfunction, and was indicated to mediate beneficial influence over adiponectin in cardiac damage induced by I/R (7-10). Adiponectin serves a vital role in the cardiac protection mechanism, the decline of which was indicated to enhance I/R injury and the ineffectiveness of ischemia post-conditioning (11). The activation of AMPK is mediated by adaptor protein phosphotyrosine interacting with $\mathrm{PH}$ domain and leucine zipper 1 (APPL1), which binds to both adiponectin receptor (AdipoR)1 and R2 (12). Adiponectin has been reported to protect myocardial cells from I/R injury by regulating AMPK and cyclooxygenase 2 (COX2) expression (13). In addition, following myocardial I/R injury induction in rats, adiponectin expression has been indicated to regulate the activation of the AMPK/sirtuin 1 axis (14). A previous study has demonstrated that adiponectin protected H9c2 cells from hypoxia-reoxygenation (H/R)-induced apoptosis, but it could also activate APPL1 (15). 
Reactive oxygen species (ROS) are the main cause of oxidative stress and the primary inducer of I/R injury (16). Under normoxic conditions, the production and clearance of ROS in myocardial cells are balanced. However, during myocardial ischemia and hypoxia, the increased production of ROS can directly result in the oxidation of lipids, proteins and DNA, which further damages the cellular structure (17). As a result, the products of lipid peroxidation and the activity levels of the antioxidant enzymes, such as superoxide dismutase (SOD), are increased, leading to myocardial injury (18). Nuclear factor erythroid 2-related factor 2 (NRF2) is involved in the regulation of redox balance, stress response and inflammation (19). It has been reported that NRF2 mediated the modulation of oxidative stress in cerebral oxygen-glucose deprivation/reoxygenation (20). In addition, an interplay between NRF2 and NF- $\kappa \mathrm{B}$ has been demonstrated, which involved counterbalancing the activity of one another in cerebrovascular disorders (21).

Based on this evidence, the present study aimed to investigate the role and mechanism of action of APPL1 in H9c2 cells following H/R injury.

\section{Materials and methods}

Cell lines and establishment of the H/R model. H9c2 (rat myocardial cells) were purchased from The Cell Bank of Type Culture Collection of The Chinese Academy of Sciences and were routinely cultured in DMEM supplemented with $10 \%$ FBS (each, Gibco; Thermo Fisher Scientific, Inc.) at $37^{\circ} \mathrm{C}$ with $5 \% \mathrm{CO}_{2} . \mathrm{H} 9 \mathrm{c} 2$ cells were subjected to hypoxia for $24 \mathrm{~h}$ in an incubator at $37^{\circ} \mathrm{C}\left(82 \% \mathrm{~N}_{2}, 18 \% \mathrm{CO}_{2}\right.$ and $\left.<0.5 \% \mathrm{O}_{2}\right)$ and reoxygenation for $1 \mathrm{~h}$ in an incubator at $37^{\circ} \mathrm{C}\left(5 \% \mathrm{CO}_{2}\right.$ and $\left.21 \% \mathrm{O}_{2}\right)$ to simulate myocardial cell injury caused by $\mathrm{I} / \mathrm{R}$, and a $\mathrm{H} / \mathrm{R}$ model was established (H/R group). Cells in control group were routinely cultured in DMEM supplemented with $10 \%$ FBS at $37^{\circ} \mathrm{C}$ with $5 \% \mathrm{CO}_{2} . \mathrm{H} 9 \mathrm{c} 2$ cells were pretreated with a specific AMPK activator (100 $\mu \mathrm{M}$; product name, MK-3903; cat. no. GC31361; GlpBio Technology) and AMPK inhibitor (10 $\mu \mathrm{M}$; product name, compound C; cat. no. HY-13418A; MedChemExpress) for $24 \mathrm{~h}$.

Western blot analysis. H9c2 cells were exposed to specific conditions (H/R, APPL1 overexpression model, AMPK inhibitor or AMPK activator) and the cell was lysed using RIPA lysis buffer containing 1\% PMSF (Beijing Solarbio Science \& Technology Co., Ltd.) on ice for $30 \mathrm{~min}$. The supernatant was collected after centrifugation at $4^{\circ} \mathrm{C}$ for $15 \mathrm{~min}$ at $12,000 \mathrm{x} \mathrm{g}$, and the protein concentration was determined via the BCA method. Subsequently, the proteins were transferred to PVDF membranes following $12 \%$ SDS-PAGE $(50 \mu \mathrm{g}$ proteins/lane were loaded on the gel). The membranes were blocked with $5 \%$ skimmed milk powder solution for $2 \mathrm{~h}$ at room temperature. The following primary antibodies were incubated with the membranes at $4^{\circ} \mathrm{C}$ overnight: AMPK (1:1,000; cat. no. ab214425; Abcam), phosphorylated (p)-AMPK (1:1,000; cat. no. ab133448; Abcam), SOD2 (1:1,000; cat. no. ab68155; Abcam), SOD3 (1:1,000; cat. no. ab83108; Abcam), COX2 (1:1,000; cat. no. ab179800; Abcam), APPL1 (1:1,000; cat. no. ab250150; Abcam), GAPDH (1:5,000; cat. no. ab181602; Abcam), NRF2 (1:1,000; cat. no. ab89443; Abcam), Bcl2 (1:1,000; cat. no. ab196495; Abcam), heme oxygenase 1 (HO-1; 1:10,000; cat. no. ab68477; Abcam), p-liver kinase B1 (p-LKB1; cat. no. 3482; 1,1000; Cell Signaling Technology, Inc.), LKB1 (cat. no. 3047; 1,1000; Cell Signaling Technology, Inc.), p-acetyl-CoA carboxylase $\alpha$ (p-ACC; cat. no. 3661; 1,1000; Cell Signaling Technology, Inc.), ACC (cat. no. 3662; 1,1000; Cell Signaling Technology, Inc.), cleaved caspase-3 (cat. no. 9664; 1,1000; Cell Signaling Technology, Inc.), cleaved poly (ADP-ribose) polymerase (PARP; cat. no. 94885; 1,1000; Cell Signaling Technology, Inc.), NF-kB p65 (cat. no. 8242; all 1:1,000; Cell Signaling Technology, Inc.). The membranes were washed with TBST and then incubated with a secondary antibody solution [goat anti-rabbit IgG H\&L (HRP); 1:10,000; cat. no. ab97051; Abcam] for $2 \mathrm{~h}$ at room temperature. Following incubation, a chemiluminescence detection kit kit (Advansta, Inc.) was used to visualize the proteins. Quantity One software (v4.6.6; Bio-Rad Laboratories, Inc.) was used to analyze the gray scale of the protein bands. The ratio of gray value of the target protein was normalized to GAPDH to represent the relative expression levels of the target protein.

SOD activity. H9c2 cells were collected and lysed using RIPA lysis buffer (Beijing Solarbio Science \& Technology, Co., Ltd.). The cell supernatant was then obtained following centrifugation at $3,000 \mathrm{xg}$ for $15 \mathrm{~min}$ at $4^{\circ} \mathrm{C}$. SOD activity was detected according to the manufacturer's protocol of the SOD assay kit (cat. no. A001-3-2; WST-1 method; Nanjing Jiancheng Bioengineering Institute). The absorbance at a wavelength of $450 \mathrm{~nm}$ was detected using a microplate reader.

Plasmid transfection. $\mathrm{H} 9 \mathrm{c} 2$ cells $\left(10 \times 10^{4}\right.$ cells $\left./ \mathrm{ml}\right)$ were transfected with APPL1 overexpression plasmids $(5 \mathrm{nM}$; ov-APPL1; Genomeditech) or empty plasmids (5 nM; pcDNA3.1; Genomeditech) using Lipofectamine ${ }^{\circledR} 3000$ reagent (Invitrogen; Thermo Fisher Scientific, Inc.) according to the manufacturer's protocol. At $24 \mathrm{~h}$ after transfection, the cells were incubated at $37^{\circ} \mathrm{C}$ for $12 \mathrm{~h}$ and then exposed to $\mathrm{H} / \mathrm{R}$ conditions, in which $\mathrm{H} 9 \mathrm{c} 2$ cells were subjected to hypoxia for $24 \mathrm{~h}$ in an incubator at $37^{\circ} \mathrm{C}\left(82 \% \mathrm{~N}_{2}, 18 \% \mathrm{CO}_{2}\right.$ and $\left.<0.5 \% \mathrm{O}_{2}\right)$ followed by reoxygenation for $1 \mathrm{~h}$ in an incubator at $37^{\circ} \mathrm{C}$ $\left(5 \% \mathrm{CO}_{2}, 21 \% \mathrm{O}_{2}\right)$ to simulate myocardial cell injury).

Reverse transcription-quantitative PCR (RT-qPCR). After $\mathrm{H} / \mathrm{R}$ induction, H9c2 cells were collected for RT-qPCR analysis. Total RNA was extracted with TRIzol ${ }^{\circledR}$ reagent (Invitrogen; Thermo Fisher Scientific, Inc.) and its concentration was measured at a wavelength of $260 \mathrm{~nm}$ by spectrophotometry. In accordance with their respective manufacturer's protocols, a high-Capacity cDNA Reverse Transcription kit (Thermo Fisher Scientific, Inc.) and SYBR $^{\mathrm{TM}}$ Green quantitative kit (Thermo Fisher Scientific, Inc.) were used to reverse transcribe RNA into cDNA and for qPCR analysis, respectively, and GAPDH was used as the internal reference. The primers used in the present study were as follows: APPL1 forward, 5'-GCCCGCAGACAAGGTCTT TA-3' and reverse, 5'-TGAGGTCAGGTGTGTTGCTG-3'; TNF- $\alpha$ forward, 5'-TGAGCACAGAAAGCATGATC-3' and reverse, 5'-CATCTGCTGGTACCACCAGTT-3'; monocyte chemoattractant protein 1 (MCP-1) forward, 5'-TCCACC 
A

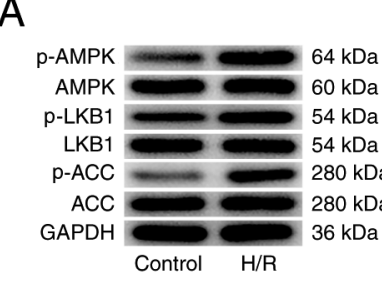

B

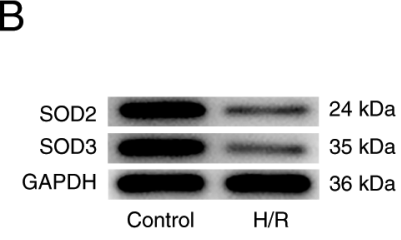

D

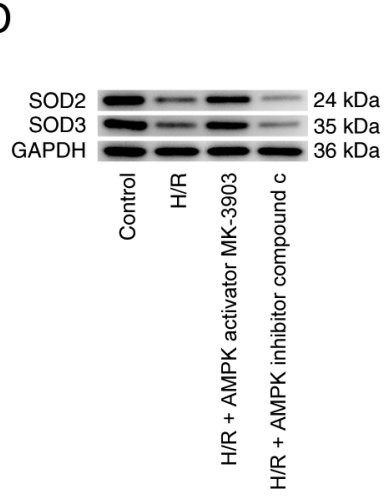

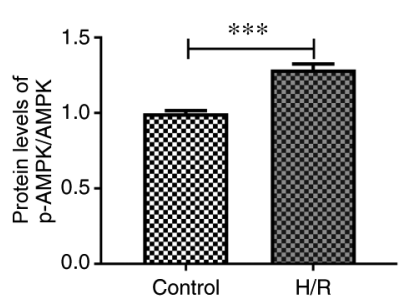
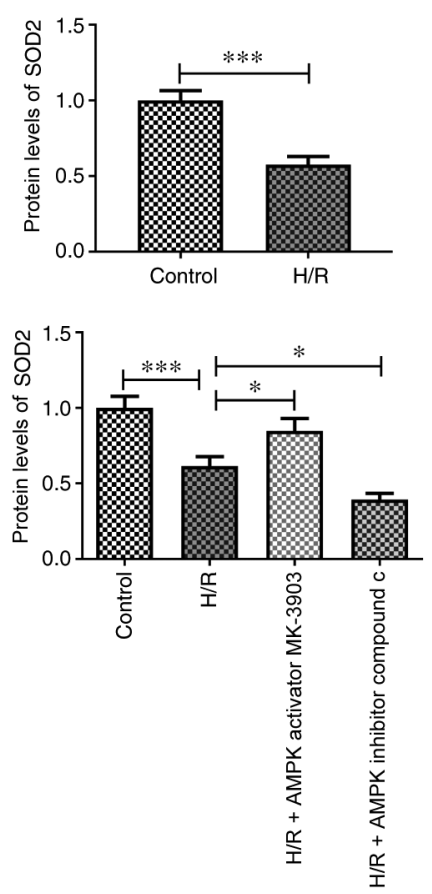
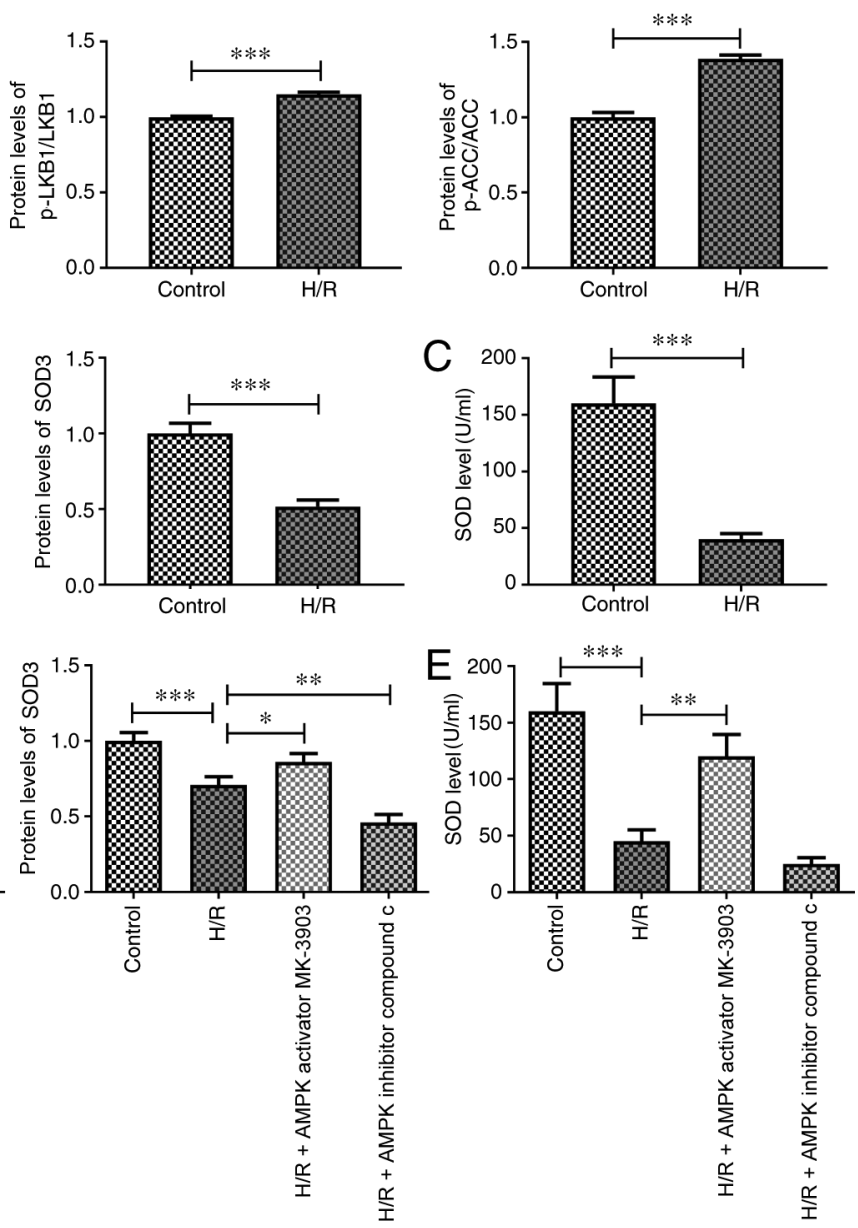

Figure 1. AMPK affects the protein expression levels of AMPK signaling and SOD levels. (A) H/R induction increased the ratios of p-AMPK/AMPK, p-LKB1/LKB1 and p-ACC/ACC. (B) Expression of SOD2 and SOD3 proteins after H/R. (C) Analysis of SOD activity levels using a SOD assay kit. (D) Analysis of SOD2 and SOD3 protein expression levels via western blotting. (E) The detection of SOD activities by SOD assay kit. The experimental data are presented as the mean $\pm \mathrm{SD}$. ${ }^{*} \mathrm{P}<0.05,{ }^{* *} \mathrm{P}<0.01$ and ${ }^{* * * *} \mathrm{P}<0.001$. SOD, superoxide dismutase; H/R, hypoxia-reoxygenation; ACC, acetyl-CoA carboxylase; AMPK, AMP-activated protein kinase; p, phosphorylated; LKB1, liver kinase B1.

ACTATGCAGGTCTC-3' and reverse, 5'-TGGACCCATTCC TTATTGGG-3'; IL-1 $\beta$, forward, 5'-GACCTGTTCTTTGAG GCTGAC-3', and reverse, 5'-TCCATCTTCTTCTTTGGG TATTGTT-3'; and GAPDH forward, 5'-AGGGGCCATCCA CAGTCTTC-3' and reverse, 5'-CAGTGCCAGCCTCGTCTC AT-3'. The qPCR thermocycling conditions were as follows: Initial denaturation at $94^{\circ} \mathrm{C}$ for $3 \mathrm{~min}$; followed by 34 cycles of annealing at $55^{\circ} \mathrm{C}$ for $45 \mathrm{sec}$ and extension at $72^{\circ} \mathrm{C}$ for $2 \mathrm{~min}$; and a final extension at $72^{\circ} \mathrm{C}$ for $7 \mathrm{~min}$. The results of the experiments were analyzed using the $2^{-\Delta \Delta \mathrm{Cq}}$ method (22).

Cell counting kit-8 (CCK-8) assay. H9c2 cells were seeded into 96 -well plates $\left(1 \times 10^{7}\right.$ cells $\left./ \mathrm{ml}\right)$. A total of $100 \mu \mathrm{l}$ cell suspension was added to each well. Following incubation, $10 \mu \mathrm{l}$ CCK-8 solution (GlpBio Technology) was added to each well and incubated for $2 \mathrm{~h}$ at $37^{\circ} \mathrm{C}$. The absorbance was measured at a wavelength of $450 \mathrm{~nm}$ with a microplate reader (Thermo Fisher Scientific, Inc.).

Lactate dehydrogenase ( $L D H$ ) assay. When cells undergo apoptosis or necrosis, the cell membrane ruptures and intracellular LDH is released into the medium (23). The cell supernatant was collected after centrifugation at $400 \mathrm{x} g$ at $4^{\circ} \mathrm{C}$ for 5 min and LDH activity was detected using a LDH kit (cat. no. ab65393; Abcam) according to the manufacturer's instructions. The absorbance value of each well was measured at a wavelength of $450 \mathrm{~nm}$ using a microplate reader, and the activity levels of $\mathrm{LDH}$ were quantitatively detected based on pyruvate levels. During the experiment, three wells were used per experimental condition.

ROS staining. $\mathrm{H} 9 \mathrm{c} 2$ cells $\left(1 \times 10^{6}\right.$ cells/well $)$ were seeded into 6-well plates and treated with $\mathrm{H} / \mathrm{R}$ (three wells for each experimental condition). A total of $1 \mu 1$ ROS probe (cat. no. HY-D0940; MedChemExpress) was added for a $30 \mathrm{~min}$ incubation at $37^{\circ} \mathrm{C}$. The culture medium was discarded, and the cells were rinsed twice with PBS. Four random fields of view were selected and imaged using a fluorescence microscope (Olympus Corporation; magnification, x200). The fluorescence intensity was detected using ImageJ $1.52 \mathrm{r}$ software (National Institutes of Health).

TUNEL assay. The apoptotic cells were stained using the TUNEL kit (cat. no. C1086; Beyotime Institute of Biotechnology) according to the manufacturer's instructions. H9c2 cells were collected, washed with PBS and 
A

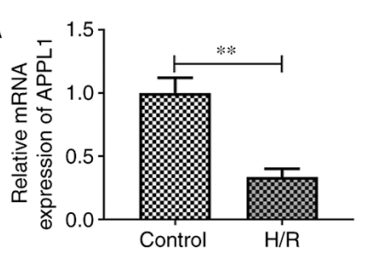

C

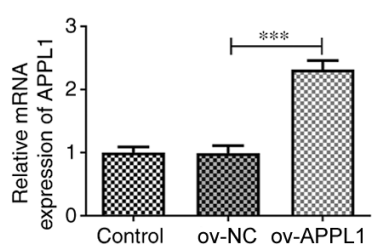

E

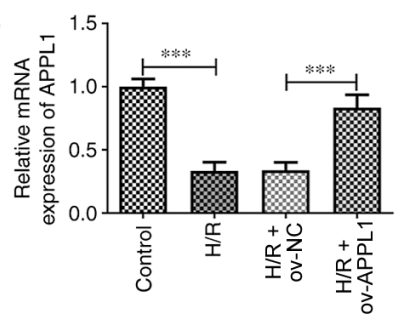

B

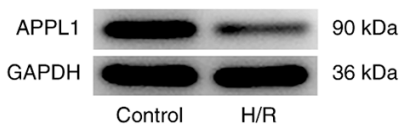

D

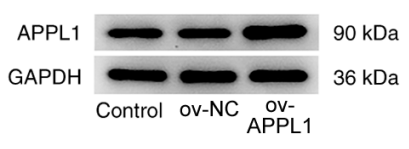

F

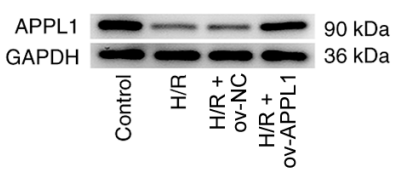

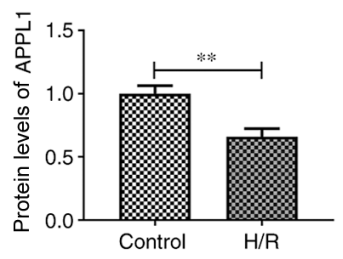
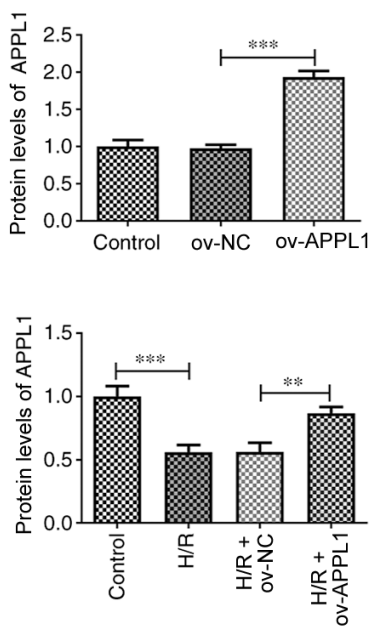

G
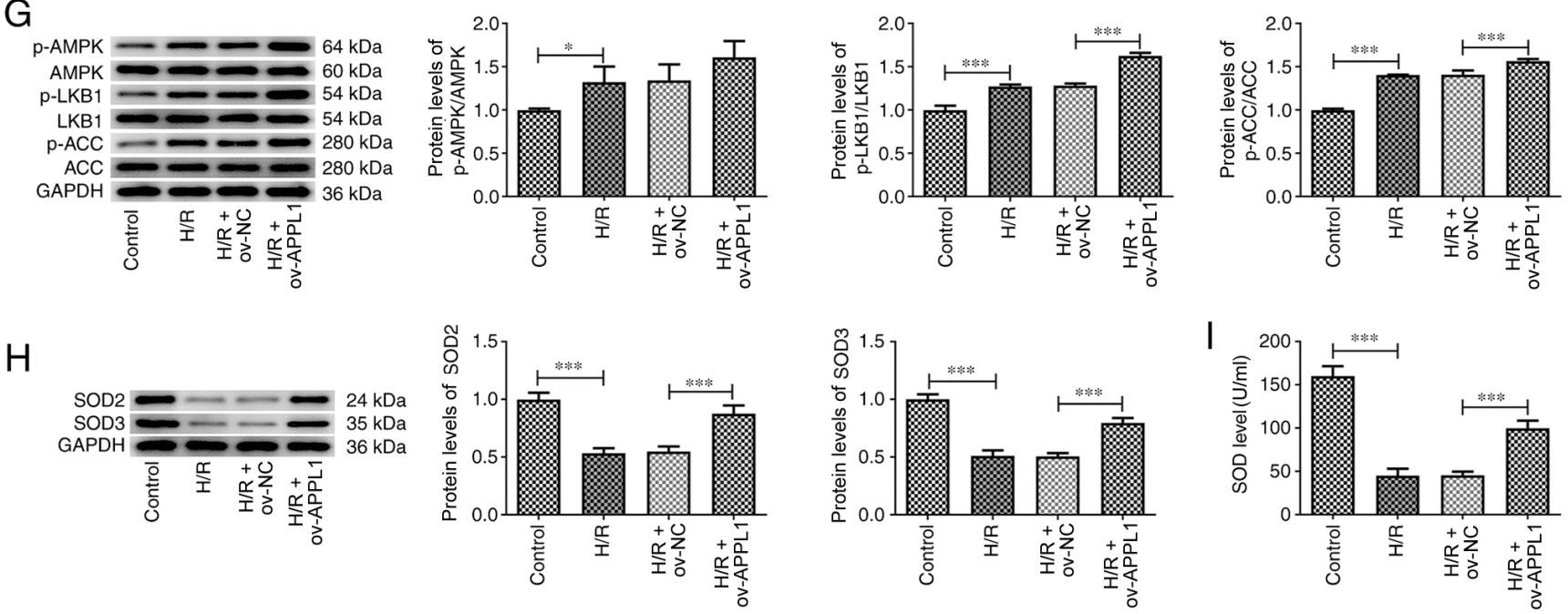

Figure 2. APPL1 overexpression increases the phosphorylation levels of LKB1, AMPK and ACC, as well as SOD levels. (A) mRNA and (B) protein expression levels of APPL1 in H/R-induced H9c2 cells. (C) mRNA and (D) protein expression levels of APPL1 in H9c2 cells with or without ov-APPL1 transfection. (E) mRNA and (F) protein expression levels of APPL1 in H/R-induced H9c2 cells overexpressing APPL1. Determination of the expression levels of (G) p-LKB1, LKB1, p-AMPK, AMPK, p-ACC, ACC, (H) SOD2 and SOD3. (I) Determination of the activity levels of SOD. The experimental data are presented as the mean $\pm \mathrm{SD}$. ${ }^{*} \mathrm{P}<0.05,{ }^{* * *} \mathrm{P}<0.01$ and ${ }^{* * *} \mathrm{P}<0.001$. APPL1, adaptor protein phosphotyrosine interacting with PH domain and leucine zipper 1 ; ACC, acetyl-CoA carboxylase; H/R, hypoxia-reoxygenation; SOD, superoxide dismutase; LKB1, liver kinase B1; AMPK, AMP-activated protein kinase; p, phosphorylated; ov, overexpression; NC, negative control.

subsequently fixed using $4 \%$ paraformaldehyde at room temperature for $30 \mathrm{~min}$. Following incubation with $0.3 \%$ Triton X-100 for $5 \mathrm{~min}$ at room temperature, the cells were further incubated with TUNEL solution at $37^{\circ} \mathrm{C}$ for $60 \mathrm{~min}$. DAPI $(0.1 \mu \mathrm{g} / \mathrm{ml})$ was added to counterstain nuclei at room temperature in the dark for $5 \mathrm{~min}$. Four random fields were selected for analysis and the apoptotic cells was observed using a fluorescence microscope (Olympus Corporation; magnification, x200).

Statistical analysis. SPSS 22.1 software (IBM Corp.) was used for analysis of all data. GraphPad Prism 7.0 (GraphPad
Software, Inc.) was used for production of the relevant graphs. The data are presented as the mean \pm SD. Each experiment was at least repeated three times. One-way ANOVA was used for comparisons among multiple groups, followed by Tukey's post hoc test. $\mathrm{P}<0.05$ was considered to indicate a statistically significant difference.

\section{Results}

$H / R$ affects the AMPK signaling pathway and the expression levels of antioxidant enzymes. AMPK activation has been reported to serve a protective role in decreasing the death 

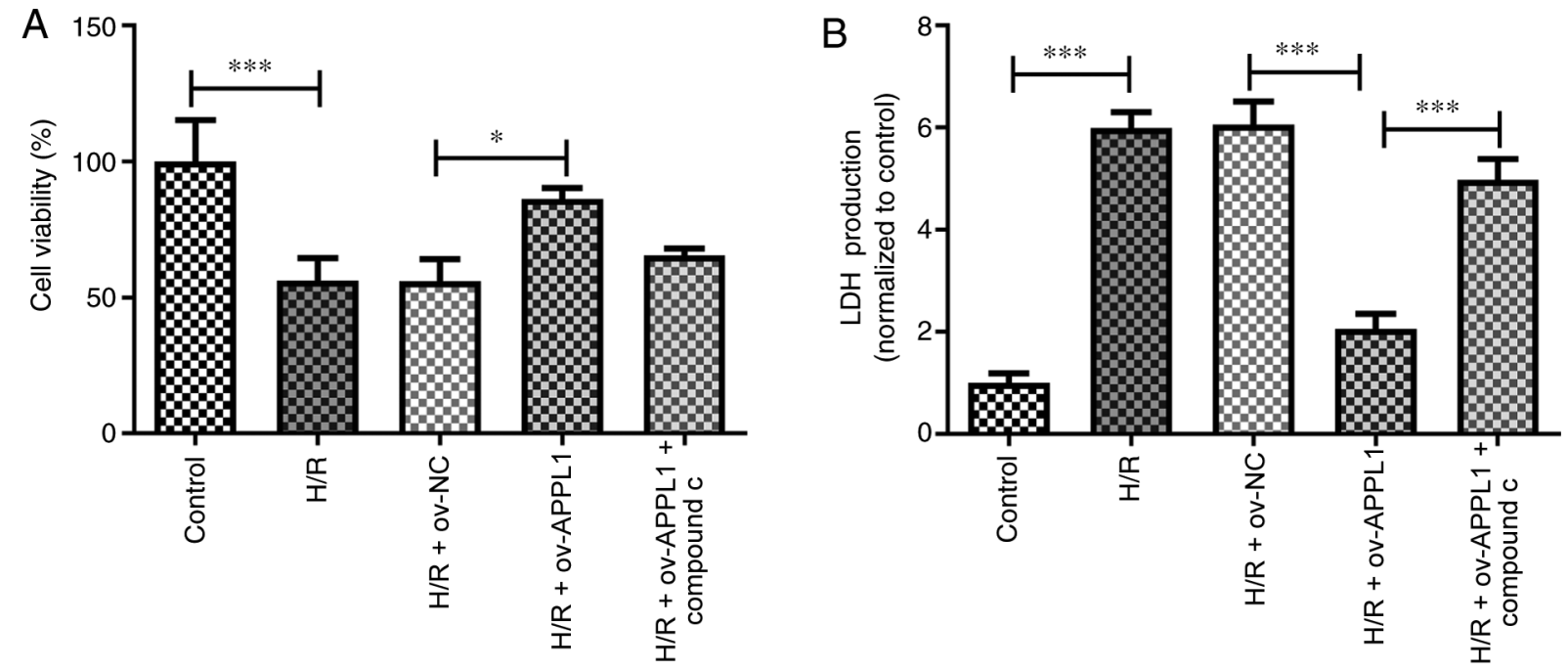

Figure 3. APPL1 overexpression increases cell viability after H/R and compound $\mathrm{C}$ reduces it via the AMP-activated protein kinase signaling pathway. (A) Cell viability and (B) LDH were detected using Cell Counting Kit- 8 and LDH assays, respectively. The experimental data are presented as the mean \pm SD. ${ }^{*} \mathrm{P}<0.05$ and ${ }^{* * *} \mathrm{P}<0.001$. APPL1, adaptor protein phosphotyrosine interacting with PH domain and leucine zipper 1; LDH, lactate dehydrogenase; H/R, hypoxia-reoxygenation; ov, overexpression; NC, negative control.
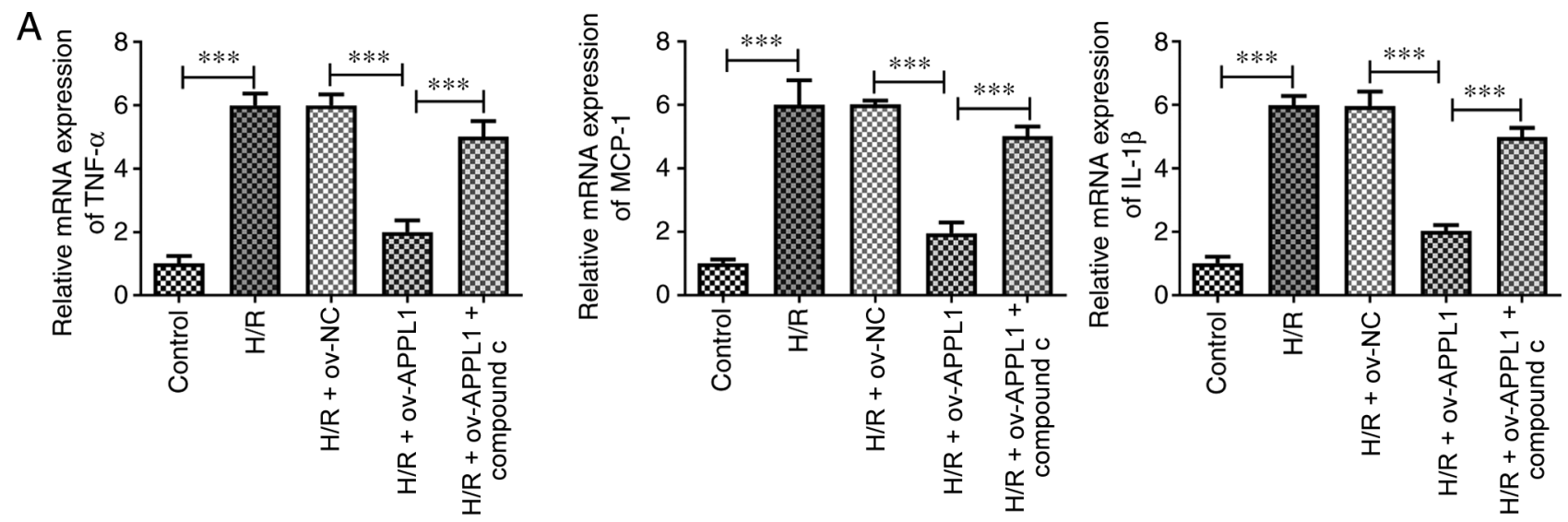

B
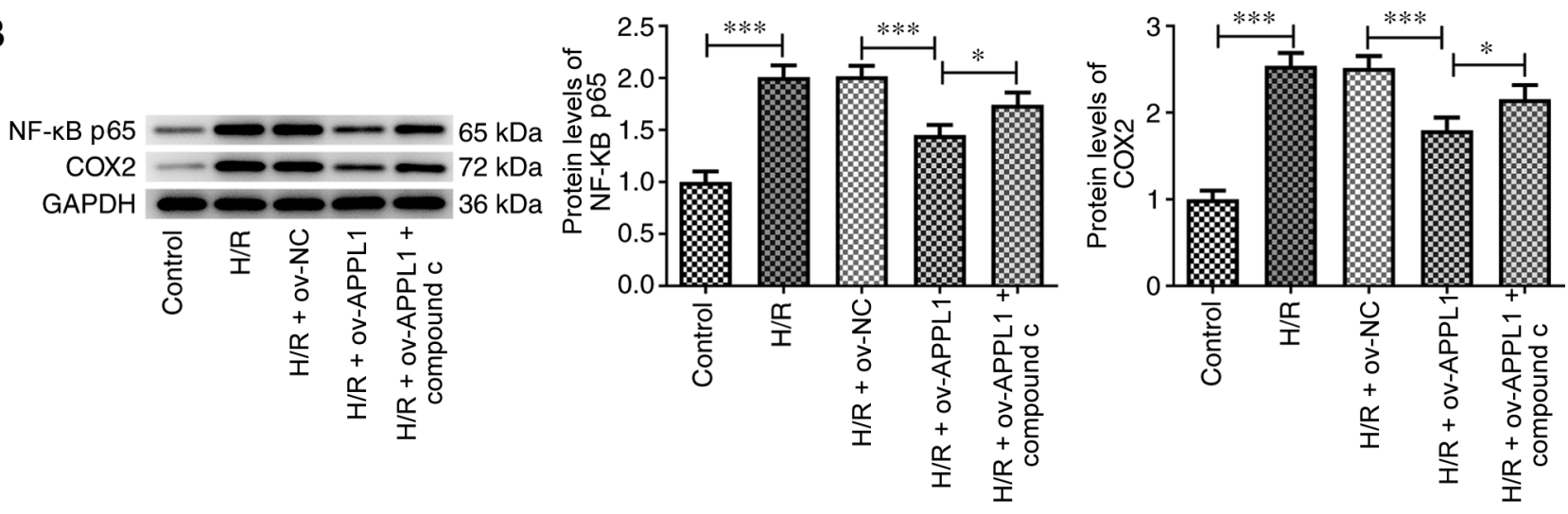

Figure 4. APPL1-induced suppression of proinflammatory mediators after $\mathrm{H} / \mathrm{R}$ is mediated by AMP-activated protein kinase. (A) Detection of the expression levels of TNF- $\alpha$, MCP-1 and IL-1 $\beta$ in H/R-induced H9c2 cells. (B) Expression levels of NF- $\mathrm{kB}$ p 65 and COX2 were determined by western blotting. The experimental data are presented as the mean $\pm \mathrm{SD}$. ${ }^{*} \mathrm{P}<0.05$ and ${ }^{* * * *} \mathrm{P}<0.001$. APPL1, adaptor protein phosphotyrosine interacting with $\mathrm{PH}$ domain and leucine zipper 1; H/R, hypoxia-reoxygenation; MCP-1, monocyte chemoattractant protein 1; COX2, cyclooxygenase 2; ov, overexpression; NC, negative control.

of myocardial cells induced by $\mathrm{I} / \mathrm{R}(4,24)$. The expression levels of the total and phosphorylated forms of I/R-associated proteins, including AMPK, LKB1 and ACC, were assessed using western blot analysis. The phosphorylation levels of these proteins were significantly increased in H/R-treated H9c2 cells compared with those in the control group (Fig. 1A). Furthermore, the expression levels of SOD2 and SOD3, and SOD activity were significantly decreased by $H / R$ treatment 


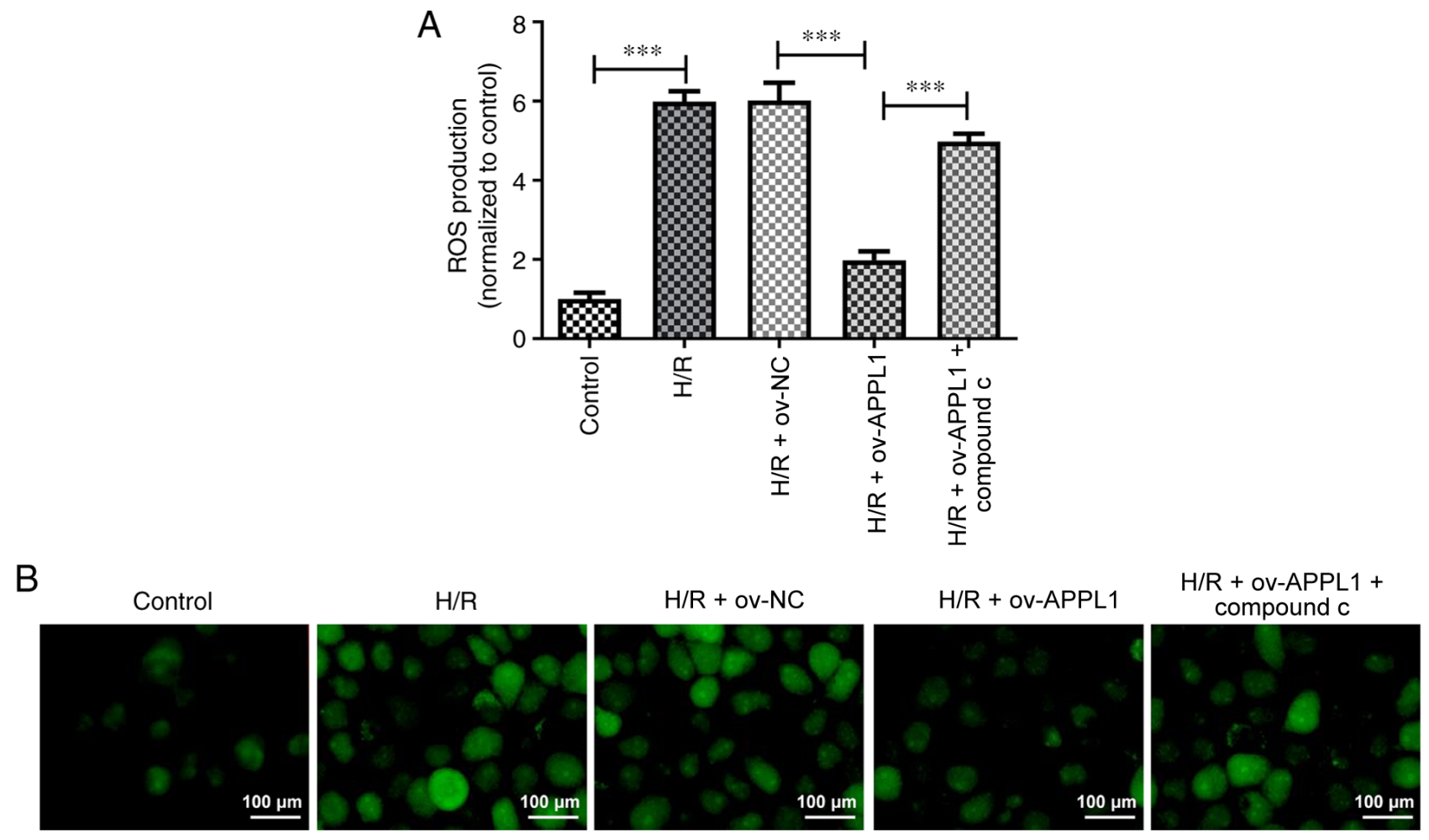

Figure 5. Decrease in ROS levels induced by APPL1 overexpression is mediated via AMP-activated protein kinase. (A) Quantification of ROS production (B) as determined by ROS staining. The experimental data are presented as the mean \pm SD. ${ }^{* * *} \mathrm{P}<0.001$. ROS, reactive oxygen species; APPL1, adaptor protein phosphotyrosine interacting with $\mathrm{PH}$ domain and leucine zipper 1; H/R, hypoxia-reoxygenation; ov, overexpression; NC, negative control.

compared with those in the control group (Fig. 1B and C). It has been reported that AMPK/GSK3 $\beta$-Nrf2 is implicated in affecting oxidative stress $(25,26)$. To verify the effects of the AMPK signaling pathway on the expression of the antioxidant enzymes, MK-3903 or compound C were added to the cells. The results indicated that the expression levels of SOD2 and SOD3, and SOD activity were tightly regulated by the AMPK signaling pathway (Fig. 1D and E).

APPL1 overexpression upregulates the expression levels of LKB1/AMPK/ACC signaling pathway-related proteins. The protein and mRNA expression levels of APPL1 were detected by western blot analysis and RT-qPCR, respectively. The results revealed that the mRNA and protein expression levels of APPL1 were significantly downregulated following $\mathrm{H} / \mathrm{R}$ exposure in $\mathrm{H} 9 \mathrm{c} 2$ cells compared with those in the control group (Fig. 2A and B). To explore the mechanism of action underlying APPL1, this protein was overexpressed in $\mathrm{H} 9 \mathrm{c} 2$ cells with or without $\mathrm{H} / \mathrm{R}$ induction (Fig. 2C-F). The phosphorylation levels of LKB1, AMPK and ACC were increased after APPL1 overexpression compared with the H/R and Ov-NC cotreatment group (Fig. 2G). In addition, the expression levels of SOD2 and SOD3, and levels of SOD activity were also significantly increased by APPL1 overexpression compared with the H/R and Ov-NC cotreatment group (Fig. 2H and I).

APPL1 increases cell viability and reduces $L D H$ release via the AMPK signaling pathway. To assess whether AMPK activation was associated with APPL1 overexpression in H/R-treated H9c2 cells, compound C was used to inhibit the activation of AMPK. Cell viability was detected using a CCK-8 assay. Following H/R treatment, the viability of H9c2 cells was significantly decreased compared with that in the control group. When the cells were transfected with ov-APPL1 plasmids for $12 \mathrm{~h}$, the results of cell viability indicated that APPL1 overexpression significantly reduced $\mathrm{H} / \mathrm{R}$-induced cell injury compared with the $\mathrm{H} / \mathrm{R}+\mathrm{ov}-\mathrm{NC}$ group, displaying no apparent cytotoxic effect (Fig. 3A). When cardiomyocytes are damaged, they release several different proteins into the bloodstream, such as LDH. The release of LDH from $\mathrm{H} 9 \mathrm{c} 2$ myocardial cells was significantly increased in the H/R group compared with that in the control group (Fig. 3B). However, the effect of APPL1 overexpression on cell viability and LDH release was significantly counteracted by inhibiting AMPK via co-treatment of the cells with compound $\mathrm{C}$.

APPL1 overexpression reduces the expression levels of proinflammatory markers via AMPK signaling. APPL1 overexpression significantly decreased the expression levels of the proinflammatory markers TNF- $\alpha$, MCP-1 and IL- $1 \beta$ in H/R-induced H9c2 cells, which was significantly reversed following compound C treatment (Fig. 4A). Similar effects were also observed after H/R treatment and APPL1 overexpression in the expression levels of p65 and COX2 (Fig. 4B), indicating that APPL1 could regulate the NF- $\mathrm{KB}$ signaling pathway via AMPK.

APPL1 overexpression decreases ROS regeneration via the $A M P K$ signaling pathway. Previous studies have indicated that $\mathrm{H} / \mathrm{R}$ can induce ROS production and oxidative damage, 
A

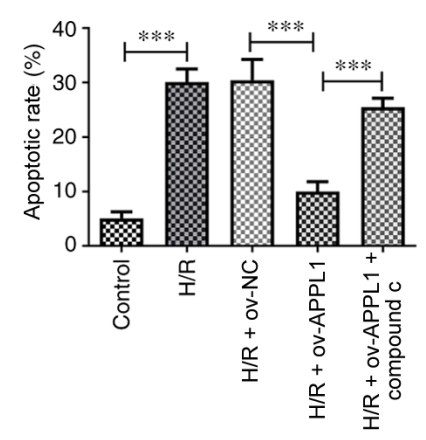

B
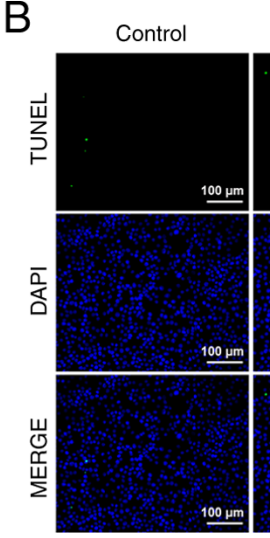

$H / R$

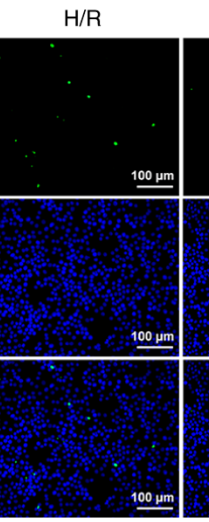

$\mathrm{H} / \mathrm{R}+$ ov-APPL1 + compound $\mathrm{c}$

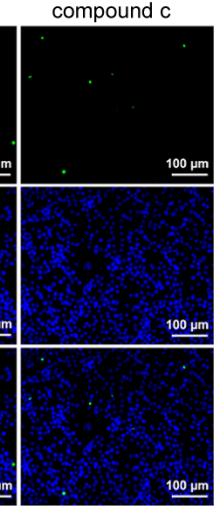

C
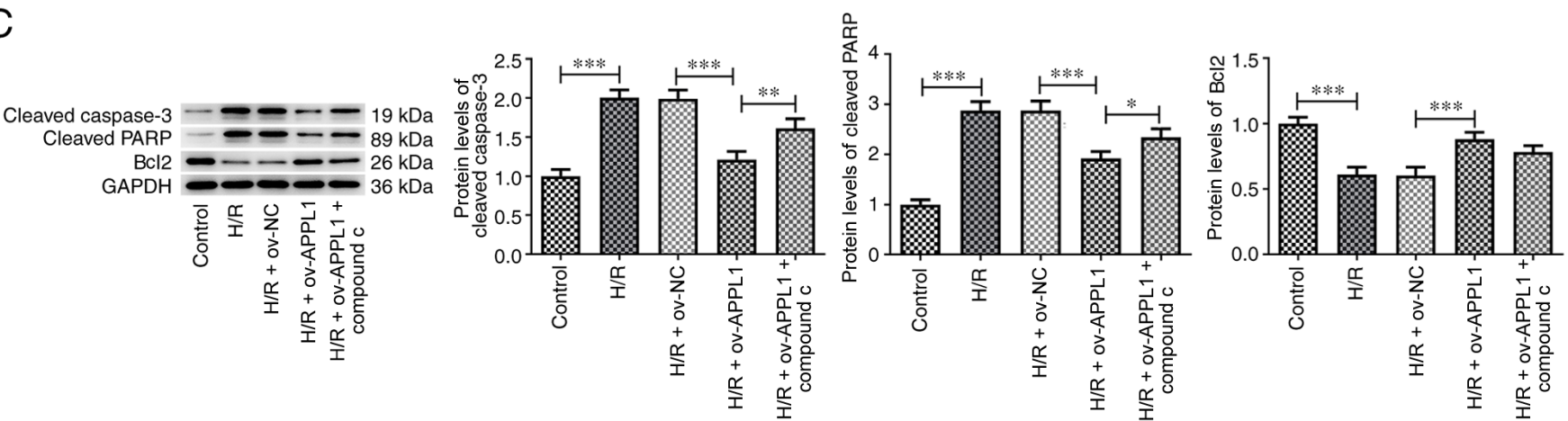

Figure 6. Suppression of apoptosis by APPL1 overexpression. (A) Quantification of apoptotic rate of H9c2 cells induced by H/R (B) as determined by performing TUNEL staining. (C) Detection of cleaved caspase-3, cleaved PARP and Bcl2 expression levels. The experimental data are presented as the mean \pm SD. ${ }^{*} \mathrm{P}<0.05,{ }^{* *} \mathrm{P}<0.01$ and ${ }^{* * * *} \mathrm{P}<0.001$. APPL1, adaptor protein phosphotyrosine interacting with $\mathrm{PH}$ domain and leucine zipper $1 ; \mathrm{H} / \mathrm{R}$, hypoxia-reoxygenation; PARP, poly (ADP-ribose) polymerase 1; ov, overexpression; NC, negative control.
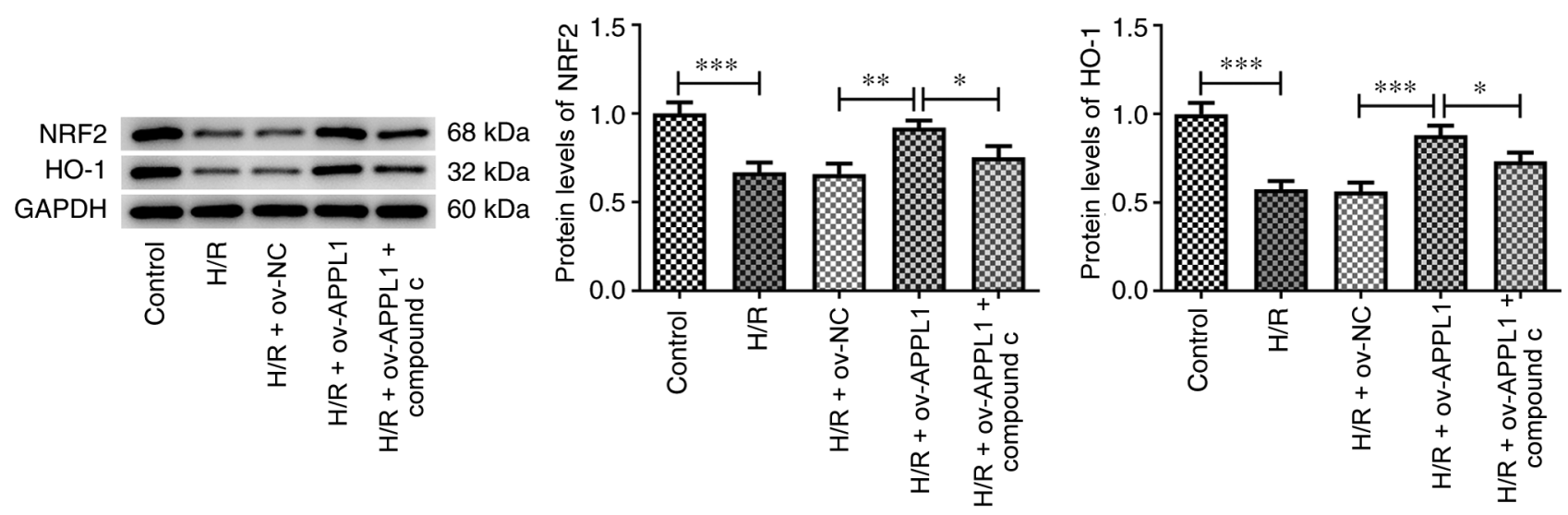

Figure 7. Upregulation of NRF2 expression by APPL1 overexpression after H/R. Expression levels of NRF2 and HO-1 in H/R-induced H9c2 cells were detected using western blotting. The experimental data are presented as the mean $\pm \mathrm{SD}$. ${ }^{*} \mathrm{P}<0.05,{ }^{* *} \mathrm{P}<0.01$ and ${ }^{* * * *} \mathrm{P}<0.001$. H/R, hypoxia-reoxygenation; $\mathrm{NRF} 2$, nuclear factor erythroid 2-related factor 2; HO-1, heme oxygenase-1; APPL1, adaptor protein phosphotyrosine interacting with PH domain and leucine zipper 1; ov, overexpression; NC, negative control.

leading to cardiomyocyte death and apoptosis $(27,28)$. In the present study, it was further revealed that ROS production was significantly decreased by APPL1 overexpression in $\mathrm{H} / \mathrm{R}$-induced H9c2 cells, as determined by ROS staining (Fig. 5A and B). However, this effect was significantly inhibited by compound $\mathrm{C}$ treatment.

APPL1 overexpression suppresses cell apoptosis induced by $H / R$. TUNEL staining was used to detect the apoptosis of $\mathrm{H} 9 \mathrm{c} 2$ cells. The apoptotic rate of the ov-APPL1 group was significantly reduced compared with that of the $H / R+$ ov-NC group. Western blot analysis was used to detect the expression levels of apoptosis-associated proteins. The data demonstrated that the expression levels of cleaved caspase-3 and cleaved PARP were significantly reduced, whereas the expression levels of $\mathrm{Bcl} 2$ were significantly increased following APPL1 overexpression in H/R-induced $\mathrm{H} 9 \mathrm{c} 2$ cells. This effect was reversed by compound $\mathrm{C}$ treatment (Fig. 6C); however, no effect was observed on Bcl-2 expression. 
APPL1 overexpression upregulates the expression of NRF2. Previous studies have reported that the activation of AMPK causes a further activation of the NRF2 signaling pathway and protects cells from oxidative damage via NRF2 $(26,29)$. Therefore, the current study assessed whether APPL1 could activate the NRF2 signaling pathway in H/R-induced myocardial cells via AMPK signaling. Nrf2 could directly regulate the promoter activities of HO-1, a vital antioxidase that is involved in myocardial ischemia reperfusion injury. Compared with those in the control group, the expression levels of NRF2 and HO-1 (30) were significantly reduced by H/R treatment and these effects were significantly recovered by APPL1 overexpression (Fig. 7). However, compound $\mathrm{C}$ significantly inhibited the effects of APPL1 overexpression in H/R-induced H9c2 cells. These results indicated that APPL1 activated the AMPK signaling pathway, thereby enhancing NRF2 signaling.

\section{Discussion}

The activation of ACC catalyzes the synthesis of malonyl CoA, which induces the transport of long chain fatty acyl-CoA into the mitochondria for $\beta$-oxidation. This process can be modulated by AMPK (31). The AMPK/ACC signaling pathway is markedly altered in myocardial I/R as reported by previous studies $(32,33)$. The present study provided evidence of this in H9c2 cells exposed to $H / R$ conditions, which was in agreement with the aforementioned studies. The phosphorylation levels of AMPK, LKB1 and ACC were markedly increased following overexpression of APPL1. The activation of the AMPK signaling pathway has been reported to mediate the cardioprotective effects of carvedilol (33). Therefore, APPL1 overexpression was indicated to serve a protective role in HR-induced H9c2 cells. Further experimental results from the present study confirmed that SOD2 and SOD3 protein expression levels were increased along with increased cell viability and decreased LDH production following overexpression of APPL1 in H/R-induced H9c2 cells. It has also been previously demonstrated that SOD reduces ROS generation induced by myocardial I/R and decreases oxidative stress-induced injury $(34,35)$.

During myocardial I/R, the number of $\mathrm{TUNEL}^{+}$cells and the levels of TNF- $\alpha, \mathrm{MCP}-1$ and IL- $1 \beta$ are notably increased (34). In the present study, this effect was also demonstrated in $\mathrm{H} 9 \mathrm{c} 2$ cells induced by $\mathrm{H} / \mathrm{R}$, which was significantly inhibited by APPL1 overexpression. In addition, compound $\mathrm{C}$ reversed these effects of APPL1 overexpression in H/R-induced H9c2 cells, suggesting that the suppression of the levels of the proinflammatory markers following APPL1 overexpression was mediated by AMPK. Similar results were also observed for NF- $\mathrm{B}$ p 65 and COX2. It has been reported that the inflammatory response caused by the induction of $\mathrm{H} / \mathrm{R}$ is improved via AMPK activation, which modulates the JNK-mediated NF- $\mathrm{NB}$ signaling pathway (36). A decrease in ROS levels has been indicated to be associated with suppressing the induction of cell apoptosis and reducing the levels of apoptosis-associated markers in I/R (34). The present study demonstrated that APPL1 overexpression inhibited ROS production in $\mathrm{H} / \mathrm{R}$-induced $\mathrm{H} 9 \mathrm{c} 2$ cells, which could lead to the suppression of $\mathrm{H} / \mathrm{R}$-induced apoptosis. This effect was significantly relieved by compound $\mathrm{C}$ treatment. The activation of the Nrf2 signaling pathway serves a protective role in improving cardiac function and decreasing myocardial infarct size, which in turn coordinates the upregulation of antioxidant and anti-inflammatory cellular mechanisms (37). Moreover, in the present study, the levels of Nrf2 and HO-1 were significantly increased by APPL1 overexpression following H/R, and these effects were suppressed by compound $\mathrm{C}$ treatment, demonstrating the regulation of the Nrf2 signaling pathway by AMPK. The AMPK/Nrf2 signaling pathway is widely involved in I/R-induced injury, such as cerebral I/R and myocardial I/R (38-41). Taken together, the data indicated that APPL1 inhibited oxidative stress and H/R-induced myocardial injury via the AMPK signaling pathway. However, whether the regulatory mechanism underlying APPL1 in the AMPK signaling pathway is also present in vivo or in other cell lines after I/R or H/R still requires further investigation, which was a limitation of the present study. The present study indicated that APPL1 may be considered as a potential therapeutic target for myocardial $\mathrm{H} / \mathrm{R}$ injury.

\section{Acknowledgements}

Not applicable.

\section{Funding}

The present study was supported by the Jiangsu Provincial Key Research and Development Program (grant no. BE2018611).

\section{Availability of data and materials}

The datasets used and/or analyzed during the current study are available from the corresponding author on reasonable request.

\section{Authors' contributions}

YC, WL, TW and DZ made substantial contributions to the conception and design of the current study, performed the experiments, interpreted the data and drafted and revised the manuscript for important intellectual content. YC and DZ confirmed the authenticity of all the raw data. All authors read and approved the final manuscript.

\section{Ethics approval and consent to participate}

Not applicable.

\section{Patient consent for publication}

Not applicable.

\section{Competing interests}

The authors declare that they have no competing interests.

\section{References}

1. Chi GC, Kanter MH, Li BH, Qian L, Reading SR, Harrison TN, Jacobsen SJ, Scott RD, Cavendish JJ, Lawrence JM, et al: Trends in acute myocardial infarction by race and ethnicity. J Am Heart Assoc 9: e013542, 2020. 
2. Reed GW, Rossi JE and Cannon CP: Acute myocardial infarction. Lancet 389: 197-210, 2017.

3. Rout A, Tantry US, Novakovic M, Sukhi A and Gurbel PA: Targeted pharmacotherapy for ischemia reperfusion injury in acute myocardial infarction. Expert Opin Pharmacother 21: 1851-1865, 2020

4. Wu Y, Liu H and Wang X: Cardioprotection of pharmacological postconditioning on myocardial ischemia/reperfusion injury. Life Sci 264: 118628, 2021

5. Chang JC, Lien CF, Lee WS, Chang HR, Hsu YC, Luo YP, Jeng JR, Hsieh JC and Yang KT: Intermittent hypoxia prevents myocardial mitochondrial $\mathrm{Ca}^{2+}$ overload and cell death during ischemia/reperfusion: The role of reactive oxygen species. Cells 8: 564, 2019.

6. Paradies G, Paradies V, Ruggiero FM and Petrosillo G: Mitochondrial bioenergetics and cardiolipin alterations in myocardial ischemia-reperfusion injury: Implications for pharmacological cardioprotection. Am J Physiol Heart Circ Physiol 315: H1341-H1352, 2018.

7. Ouchi N, Shibata R and Walsh K: Cardioprotection by adiponectin. Trends Cardiovasc Med 16: 141-146, 2006.

8. Feng Y, Lu Y, Liu D, Zhang W, Liu J, Tang H and Zhu Y: Apig enin-7-O- $\beta$-d-(-6"-p-coumaroyl)-glucopyranoside pretreatment attenuates myocardial ischemia/reperfusion injury via activating AMPK signaling. Life Sci 203: 246-254, 2018.

9. Liu Z, Chen JM, Huang H, Kuznicki M, Zheng S, Sun W, Quan N, Wang L, Yang H, Guo HM, et al: The protective effect of trimetazidine on myocardial ischemia/reperfusion injury through activating AMPK and ERK signaling pathway. Metabolism 65: 122-130,2016.

10. Zhang Y, Wang Y, Xu J, Tian F, Hu S, Chen Y and Fu Z: Melatonin attenuates myocardial ischemia-reperfusion injury via improving mitochondrial fusion/mitophagy and activating the AMPK-OPA1 signaling pathways. J Pineal Res 66: e12542, 2019

11. Cao C, Liu HM, Li W, Wu Y, Leng Y, Xue R, Chen R, Tang LH Sun Q, Xia Z, et al: Role of adiponectin in diabetes myocardia ischemia-reperfusion injury and ischemic postconditioning. Acta Cir Bras 35: e202000107, 2020.

12. Fang $\mathrm{H}$ and Judd RL: Adiponectin regulation and function. Compr Physiol 8: 1031-1063,2018.

13. Shibata R, Sato K, Pimentel DR, Takemura Y, Kihara S, Ohashi K, Funahashi T, Ouchi N and Walsh K: Adiponectin protects against myocardial ischemia-reperfusion injury through AMPK- and COX-2-dependent mechanisms. Nat Med 11: 1096-1103, 2005.

14. Potenza MA, Sgarra L, Nacci C, Leo V, De Salvia MA and Montagnani M: Activation of AMPK/SIRT1 axis is required for adiponectin-mediated preconditioning on myocardial ischemia-reperfusion (I/R) injury in rats. PLoS One 14: e0210654, 2019.

15. Park M, Youn B, Zheng XL, Wu D, Xu A and Sweeney G: Globular adiponectin, acting via AdipoR1/APPL1, protects H9c2 cells from hypoxia/reoxygenation-induced apoptosis. PLoS One 6: e19143,2011.

16. Yi W, Sun Y, Gao E, Wei X, Lau WB, Zheng Q, Wang Y, Yuan Y, Wang X, Tao L, et al: Reduced cardioprotective action of adiponectin in high-fat diet-induced type II diabetic mice and its underlying mechanisms. Antioxid Redox Signal 15: 1779-1788, 2011.

17. Farías JG, Molina VM, Carrasco RA, Zepeda AB, Figueroa E, Letelier P and Castillo RL: Antioxidant therapeutic strategies for cardiovascular conditions associated with oxidative stress Nutrients 9: 966, 2017.

18. Kurian GA, Rajagopal R, Vedantham S and Rajesh M: The role of oxidative stress in myocardial ischemia and reperfusion injury and remodeling: Revisited. Oxid Med Cell Longev 2016: 1656450,2016

19. Gonçalves AF, Polegato BF, Fernandes AA, Ishikawa LL, Okoshi K, Bazan SGZ, Minicucci MF, Azevedo PS, Ikoma MR, Penitenti M, et al: Zinc supplementation attenuates cardiac remodeling after experimental myocardial infarction. Cell Physiol Biochem 50: 353-362, 2018.

20. Yin $\mathrm{H}, \mathrm{Wu} \mathrm{M}$ and Jia $\mathrm{Y}$ : Knockdown of IL-32 protects PC12 cells against oxygen-glucose deprivation/reoxygenation-induced injury via activation of Nrf2/NF- $\kappa \mathrm{B}$ pathway. Metab Brain Dis 35: 363-371, 2020.

21. Sivandzade F, Prasad S, Bhalerao A and Cucullo L: NRF2 and NF-KB interplay in cerebrovascular and neurodegenerative disorders: Molecular mechanisms and possible therapeutic approaches. Redox Biol 21: 101059, 2019.

22. Livak KJ and Schmittgen TD: Analysis of relative gene expression data using real-time quantitative PCR and the 2(-Delta Delta C(T)) method. Methods 25: 402-408, 2001.

23. Zhao D, Sun Y, Tan Y, Zhang Z, Hou Z, Gao C, Feng P, Zhang X, Yi W and Gao F: Short-duration swimming exercise after myocardial infarction attenuates cardiac dysfunction and regulates mitochondrial quality control in aged mice. Oxid Med Cell Longev 2018: 4079041, 2018.
24. Wang X, Yang L, Kang L, Li J, Yang L, Zhang J, Liu J, Zhu M, Zhang Q, Shen Y and Qi Z: Metformin attenuates myocardial ischemia-reperfusion injury via up-regulation of antioxidant enzymes. PLoS One 12: e0182777, 2017.

25. Lv H, Liu Q, Wen Z, Feng H, Deng X and Ci X: Xanthohumol ameliorates lipopolysaccharide (LPS)-induced acute lung injury via induction of AMPK/GSK3 $\beta$-Nrf2 signal axis. Redox Biol 12 311-324, 2017.

26. Lv H, Hong L, Tian Y, Yin $\mathrm{C}$, Zhu $\mathrm{C}$ and Feng $\mathrm{H}$ : Corilagin alleviates acetaminophen-induced hepatotoxicity via enhancing the AMPK/GSK $3 \beta-$ Nrf2 signaling pathway. Cell Commun Signal 17: 2, 2019

27. Guo W, Liu X, Li J, Shen Y, Zhou Z, Wang M, Xie Y, Feng X, Wang $\mathrm{L}$ and $\mathrm{Wu} \mathrm{X}$ : Prdx1 alleviates cardiomyocyte apoptosis through ROS-activated MAPK pathway during myocardial ischemia/reperfusion injury. Int J Biol Macromol 112: 608-615, 2018.

28. Liu C, Tang M, Zhang X, Li J and Cao G: Knockdown of miR-665 protects against cardiomyocyte ischemia/reperfusion injury-induced ros accumulation and apoptosis through the activation of Pak1/Akt signaling in myocardial infarction. Int Heart J 61: 347-354, 2020.

29. Yu J, Wang WN, Matei N, Li X, Pang JW, Mo J, Chen SP, Tang JP, Yan $\mathrm{M}$ and Zhang JH: Ezetimibe attenuates oxidative stress and neuroinflammation via the AMPK/Nrf2/TXNIP pathway after MCAO in rats. Oxid Med Cell Longev 2020: 4717258, 2020.

30. Zhao Z, Tang Z, Zhang W, Liu J, Li B and Ding S: Inactivated pseudomonas aeruginosa protects against myocardial ischemia reperfusion injury via Nrf2 and HO-1. Exp Ther Med 19: 3362-3368, 2020.

31. Folmes CD and Lopaschuk GD: Role of malonyl-CoA in heart disease and the hypothalamic control of obesity. Cardiovasc Res 73: 278-287, 2007.

32. Mu F, Duan J, Bian H, Zhai X, Shang P, Lin R, Zhao M, Hu D, Yin Y, Wen A and Xi M: Metabonomic strategy for the evaluation of chinese medicine salvia miltiorrhiza and dalbergia odorifera interfering with myocardial ischemia/reperfusion injury in rats. Rejuvenation Res 20: 263-277, 2017.

33. Hu H, Li X, Ren D, Tan Y, Chen J, Yang L, Chen R, Li J and Zhu P: The cardioprotective effects of carvedilol on ischemia and reperfusion injury by AMPK signaling pathway. Biomed Pharmacother 117: 109106, 2019

34. Bae S, Park M, Kang C, Dilmen S, Kang TH, Kang DG, Ke Q, Lee SU, Lee D and Kang PM: Hydrogen peroxide-responsive nanoparticle reduces myocardial ischemia/reperfusion injury. J Am Heart Assoc 5: e003697, 2016.

35. Hadi NR, Al-Amran F, Yousif M and Zamil ST: Antiapoptotic effect of simvastatin ameliorates myocardial ischemia/reperfusion injury. ISRN Pharmacol 2013: 815094, 2013.

36. Chen X, Li X, Zhang W, He J, Xu B, Lei B, Wang Z, Cates C, Rousselle $\mathrm{T}$ and $\mathrm{Li} \mathrm{J}$ : Activation of AMPK inhibits inflammatory response during hypoxia and reoxygenation through modulating JNK-mediated NF- $\mathrm{KB}$ pathway. Metabolism 83: 256-270, 2018

37. Shen Y, Liu X, Shi J and Wu X: Involvement of Nrf2 in myocardial ischemia and reperfusion injury. Int J Biol Macromol 125: 496-502, 2019

38. Hou X, Fu M, Cheng B, Kang Y and Xie D: Galanthamine improves myocardial ischemia-reperfusion-induced cardiac dysfunction, endoplasmic reticulum stress-related apoptosis, and myocardial fibrosis by suppressing AMPK/Nrf2 pathway in rats. Ann Transl Med 7: 634, 2019.

39. Mo Y, Zhu JL, Jiang A, Zhao J, Ye L and Han B: Compound 13 activates AMPK-Nrf2 signaling to protect neuronal cells from oxygen glucose deprivation-reoxygenation. Aging (Albany NY) 11: 12032-12042, 2019.

40. Wu WY, Li YD, Cui YK, Wu C, Hong YX, Li G, Wu Y, Jie LJ, Wang Y and Li GR: The natural flavone acacetin confers cardiomyocyte protection against hypoxia/reoxygenation injury via AMPK-mediated activation of Nrf2 signaling pathway. Front Pharmacol 9: 497, 2018.

41. Zhou F, Wang M, Ju J, Wang Y, Liu Z, Zhao X, Yan Y, Yan S, Luo X and Fang Y: Schizandrin A protects against cerebral ischemia-reperfusion injury by suppressing inflammation and oxidative stress and regulating the AMPK/Nrf2 pathway regulation. Am J Transl Res 11: 199-209, 2019.

This work is licensed under a Creative Commons Attribution-NonCommercial-NoDerivatives 4.0 International (CC BY-NC-ND 4.0) License. 